\title{
Handover of anesthesia care is associated with an increased risk of delirium in elderly after major noncardiac surgery: results of a secondary analysis
}

\author{
Guang-Yu Liu ${ }^{1} \cdot$ Xian Su$^{1} \cdot$ Zhao-Ting Meng $^{1} \cdot$ Fan Cui $^{1} \cdot$ Hong-Liang $\mathrm{Li}^{2} \cdot$ Sai-Nan Zhu ${ }^{3} \cdot$ Dong-Xin Wang ${ }^{1}$ (D)
}

Received: 10 January 2019 / Accepted: 22 February 2019 / Published online: 28 February 2019

(c) The Author(s) 2019

\begin{abstract}
In patients undergoing major surgery, complete handover of intraoperative anesthesia care is associated with adverse postoperative outcomes including high mortality and more major complications. The purpose of this study was to explore the association between the intraoperative complete handover between anesthesiologists and the occurrence of postoperative delirium. This was a secondary analysis of the database of a previously published clinical trial. Seven hundred patients aged 65 years or older, who were admitted to the intensive care unit after noncardiac surgery, were included. Delirium was assessed with the Confusion Assessment Method for the Intensive Care Unit twice daily during the first 7 postoperative days. Other postoperative outcomes were also monitored. The association between the intraoperative complete handover of anesthesia care and the development of postoperative delirium was analyzed with a logistic regression model. Of the 700 enrolled patients, 111 (15.9\%) developed postoperative delirium within 7 days. After correction for confounding factors, intraoperative complete handover between anesthesiologists was associated with an increased risk of postoperative delirium (OR 1.787, 95\% CI 1.012-3.155, $P=0.046$ ). Patients with intraoperative complete handover also had higher incidence of non-delirium complications $(P=0.003)$ and stayed longer in hospital after surgery $(P=0.002)$. For elderly patients admitted to the intensive care unit after noncardiac surgery, intraoperative complete handover of anesthesia care was associated with an increased risk of postoperative delirium. Chinese Clinical Trial Registry (http://www.chictr.org.cn): ChiCTR-TRC-10000802.
\end{abstract}

Keywords Elderly $\cdot$ Noncardiac surgery $\cdot$ Handover $\cdot$ Postoperative outcome $\cdot$ Delirium

\section{Introduction}

Delirium is an acutely occurring cerebral dysfunction characterized with transient and fluctuating disturbances in attention, consciousness and cognition. It is a common

Electronic supplementary material The online version of this article (https://doi.org/10.1007/s00540-019-02627-3) contains supplementary material, which is available to authorized users.

Dong-Xin Wang

wangdongxin@hotmail.com

1 Department of Anesthesiology and Critical Care Medicine, Peking University First Hospital, No. 8 Xishiku Street, Beijing 100034, China

2 Department of Critical Care Medicine, Peking University Third Hospital, Beijing, China

3 Department of Biostatistics, Peking University First Hospital, Beijing, China complication in the elderly after surgery, with reported incidence varying from 3.6 to $54.4 \%$ [1-3]. Delirium development is associated with worse outcomes, including prolonged hospital stay, increased medical costs, elevated readmission rates, declined quality of life, and shortened long-term survival [4-6]. The occurrence of postoperative delirium is a result of the interaction of multiple factors, including predisposing and precipitating factors [4, 6-8]. Being greater than 65 years old and admission to intensive care unit (ICU) are significant risk factors, and patients with these characteristics may have a delirium incidence up to $87 \%$ [9].

With the aging population and the increasing number of surgical cases $[10,11]$, intraoperative handover of anesthesia care is inevitable in some cases due to personal problem, such as fatigue or illness, or department commitments [12]. Handover can be temporary (initial anesthesiologist returns after a break) or complete (initial anesthesiologist no longer returns) [13]. After complete handovers, the incoming 
anesthesiologist receives all the information in a busy environment with many distractions. Studies showed that a high proportion of intraoperative handover between anesthesiologists is insufficient [14], and that complete handover of anesthesia care is associated with worse outcomes, including increased all-cause death and major complications within 30 days after surgery [13]. However, the impact of anesthesia handover on postoperative delirium remains unclear.

The purpose of this secondary analysis was to analyze the association between complete handover of anesthesia care and the risk of delirium development in elderly patients who were admitted to ICU after noncardiac surgery.

\section{Methods}

\section{Study design}

This was a secondary analysis of the database of a previously published clinical trial [15]. The purpose of the original trial was to explore the role of dexmedetomidine in preventing postoperative delirium in critically ill elderly patients. The study protocol was approved by the Clinical Research Ethics Committee of Peking University First Hospital (number 2011 [10]) and registered with Chinese Clinical Trial Registry (http://www.chictr.org.cn, number ChiCTR-TRC-10000802). The original study was conducted from August 17, 2011 to November 20, 2013 in the ICUs of Peking University First Hospital and Peking University Third Hospital. Written informed consents were obtained from patients, their next of kin or their legal representatives. Approval from the ethics committee for this secondary analysis was waived because the analysis was based on completely anonymized data without personal identification code. The privacy of participants was strictly observed.

\section{Patients}

The inclusion criteria were elderly patients (age $\geq 65$ years) who were admitted to the ICU after elective noncardiac surgery under general anesthesia. Patients who met any of the following criteria were excluded: (1) preoperative history of schizophrenia, epilepsy, Parkinson's disease, or myasthenia gravis; (2) inability to communicate because of coma, severe dementia or language barriers before surgery; (3) brain trauma or neurosurgery; (4) preoperative left ventricular ejection fraction (LVEF) $<30 \%$, sick sinus syndrome, heart rate $<50$ beats/min or two degree or higher atrioventricular block without pacemaker; (5) severe liver dysfunction (Child-Pugh C grade) or severe renal dysfunction (preoperative renal replacement therapy); or (6) expected survival $\leq 24 \mathrm{~h}$.

\section{Handover of patient care}

During surgery, complete handover of anesthesia care was defined as transfer of patient care from one anesthesiologist to another and the original anesthesiologist did not return [13]. In the participating hospitals, handover of anesthesia care occurred between 4 and $5 \mathrm{pm}$ and was marked in the electronically recorded worksheet. At the end of surgery, all patients were transferred to the ICU, and handover of patient care occurred between the anesthesiologists and the ICU physicians.

\section{Delirium assessment}

Delirium was assessed twice daily (8-10 am and 6-8 pm) during the first 7 days after surgery. This was performed with the Confusion Assessment Method for the Intensive Care Unit (CAM-ICU) in two steps [16]. First, the state of sedation/agitation was assessed using the Richmond Agitation Sedation Scale (RASS) [17]. For patients who were intubated and mechanically ventilated, sedative (propofol and/or midazolam) administration was titrated to a RASS of -2 or higher until extubation. For those who were deeply sedated or unarousable (RASS -4 to -5 ), delirium was not assessed and patients were recorded as comatose; for those with a RASS score of -3 or higher, delirium was assessed using the CAM-ICU which detected four features, i.e., (1) an acute onset or fluctuation course; (2) inattention; (3) thinking disorder; and (4) altered level of consciousness. Delirium was diagnosed when patients presented features $1,2,3$ or $1,2,4$. Investigators who performed delirium assessment were trained by a psychiatrist to use the CAM-ICU before the study period.

\section{Data collection}

Demographic information included gender, age, body mass index (BMI), and years of education. Baseline data included admission diagnosis, preoperative comorbidities, previous history of surgery, preoperative laboratory test results, and the American Society of Anesthesiologists (ASA) classification. Intraoperative data included method and duration of anesthesia, use of anesthetics and analgesics, use of glucocorticoids, type and duration of surgery, estimated blood loss, as well as fluid infusion and blood transfusion. Grade of surgery is rated according to the NICE Guidance of routine preoperative tests for elective surgery [18]. Cardiac risk of surgery is rated according to the ACC/AHA 2007 guidelines on perioperative cardiovascular evaluation and care for noncardiac surgery [19]. Postoperative data included type of 
patient-controlled analgesia, supplemental sedatives and analgesics, prophylactic dexmedetomidine infusion (continuous infusion at a rate of $0.1 \mu \mathrm{g} / \mathrm{kg} / \mathrm{h}$ from ICU admission to 8 am of next morning) [15], use and duration of mechanical ventilation, length of stay in ICU and hospital, occurrence of non-delirium complications within 30 days, and all-cause 30-day mortality. Non-delirium complications were generally defined as new-onset medical events other than delirium that were harmful to patients' recovery and required therapeutic intervention (Online Resource 1). Investigators who performed patient recruitment and data collection were trained before the study period.

\section{Statistical analysis}

Patients were divided into two groups according to the presence of complete anesthesia handover or not. Continuous variables were presented as mean $\pm \mathrm{SD}$ and analyzed with independent samples $t$ test or Mann-Whitney $U$ test. Categorical variables were presented as number $(\%)$ and analyzed with the Chi square test or Fisher's exact test. Time-to-event variables were presented as median $(95 \%$ confidence interval of median) analyzed with the Kaplan-Meier estimator, with differences compared with the Log-rank test. Factors that might be associated with the development of postoperative delirium were screened with univariate logistic regression analyses, and those with a $P$ value $<0.05$ were included in a multivariate logistic regression model to assess the riskadjusted association between the complete handover of anesthesia care and the development of postoperative delirium. SPSS 25.0 software package (SPSS Inc, Chicago, Ill) was used for statistical analysis. Two-sided $P$ values of less than 0.05 were considered statistically significant.

\section{Results}

\section{Patient recruitment}

From August 2011 to November 2013, 2016 patients were screened; of these, 835 were eligible, and 700 were enrolled and completed the study (Fig. 1). Compared with patients without completed handover of anesthesia care, those with complete handover were younger $(P=0.001)$, suffered less hypertension before surgery $(P=0.045)$, underwent longer anesthesia and surgery (all $P<0.001)$ and more major or complex surgery $(P=0.006)$, lost more blood but received more fluid infusion and blood transfusion during surgery (all $P<0.001$ ), and received more mechanical ventilation as well as propofol sedation during ICU stay after surgery (all $P<0.001$ ) (Tables 1, 2).

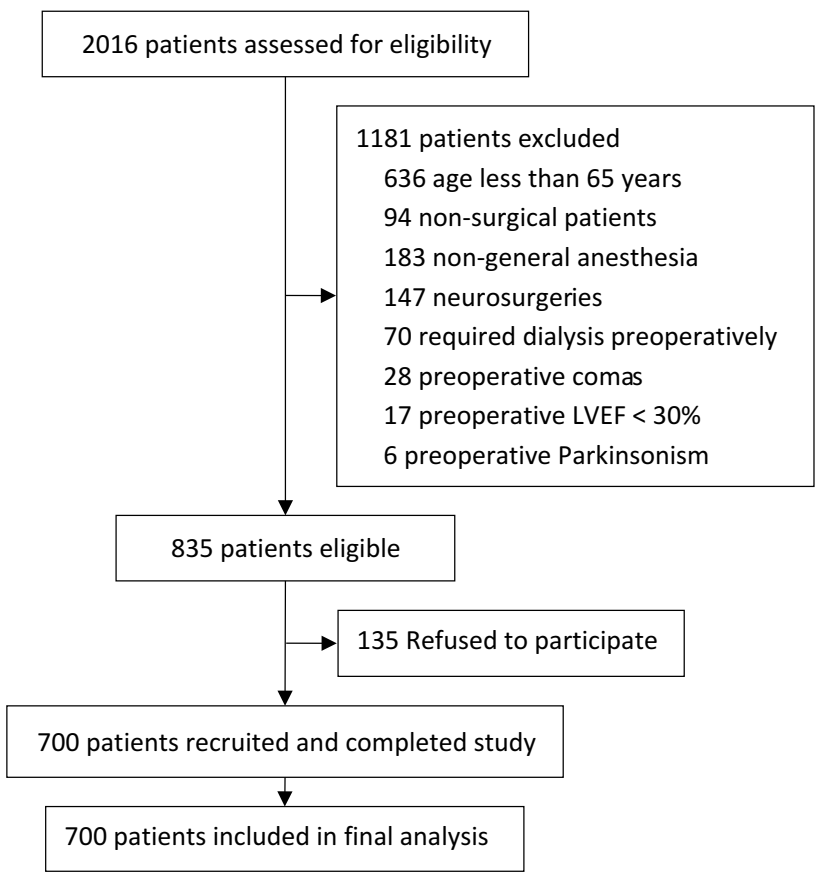

Fig. 1 Flowchart of the study

\section{Unadjusted postoperative outcomes}

Compared with patients without complete handover of anesthesia care, those with complete handover had a higher incidence of delirium within 7 days after surgery [22.6\% (23/102) vs. $14.7 \%$ (88/598), $P=0.045$ ] (Fig. 2); they also had a higher incidence of non-delirium complications within 30 days $[28.4 \%(29 / 102)$ vs. $16.0 \%(96 / 598), P=0.003]$ and stayed longer in hospital after surgery [ 14.0 days $(95 \% \mathrm{CI}$ $12.0,16.0)$ vs. 10.0 days $(9.4,10.6), P=0.002$ ] (Table 3 ; Fig. 3a, b).

\section{Association between anesthesia handover and delirium development}

Apart from complete handover of anesthesia care, univariate analyses identified nine other factors that were associated with the risk of delirium development after surgery, including age, BMI, previous stroke, preoperative albu$\min <30 \mathrm{~g} / \mathrm{L}$, intraoperative etomidate, ICU admission with intubation, prophylactic dexmedetomidine, postoperative propofol within 7 days, and pathologically diagnosed cancer (Online Resource 2). Of these, postoperative propofol within 7 days was not included in the multivariable model because it was related to ICU admission with intubation. After correction with the above confounding factors, complete handover of anesthesia care remained as an independent factor that was associated with an increased risk of postoperative delirium (OR 1.787, 95\% CI 1.012-3.155, $P=0.046$ ) (Table 4). 
Table 1 Baseline variables

\begin{tabular}{|c|c|c|c|c|}
\hline Variable & All patients $(n=700)$ & $\begin{array}{l}\text { With com- } \\
\text { plete handover } \\
(n=102)\end{array}$ & $\begin{array}{l}\text { Without complete } \\
\text { handover }(n=598)\end{array}$ & $P$ value \\
\hline Age, years & $74.3 \pm 6.8$ & $72.3 \pm 6.7$ & $74.7 \pm 6.8$ & 0.001 \\
\hline Male gender & $423(60.4)$ & $67(65.7)$ & $356(59.5)$ & 0.240 \\
\hline Body mass index, $\mathrm{kg} / \mathrm{m}^{2}$ & $23.7 \pm 3.9$ & $23.8 \pm 4.2$ & $23.7 \pm 3.9$ & 0.739 \\
\hline Education, years & $9.0(6.0,12.0)$ & $9.0(6.0,12.0)$ & $9.0(6.0,12.0)$ & 0.779 \\
\hline \multicolumn{5}{|l|}{ Preoperative comorbidity } \\
\hline Previous stroke & $161(23.0)$ & $21(20.6)$ & $140(23.4)$ & 0.531 \\
\hline Hypertension & $446(63.7)$ & $56(54.9)$ & $390(65.2)$ & 0.045 \\
\hline Coronary heart disease & $232(33.1)$ & $27(26.5)$ & $205(34.3)$ & 0.121 \\
\hline COPD & $42(6.0)$ & $5(4.9)$ & $37(6.2)$ & 0.613 \\
\hline Chronic smoking $^{\mathrm{a}}$ & $176(25.1)$ & $32(31.4)$ & $144(24.1)$ & 0.117 \\
\hline Diabetes mellitus & $190(27.1)$ & $21(20.6)$ & $169(28.3)$ & 0.107 \\
\hline Liver injury ${ }^{\mathrm{b}}$ & $19(2.7)$ & $2(2.0)$ & $17(2.8)$ & $>0.999$ \\
\hline Renal injury $^{c}$ & $35(5.0)$ & $4(3.9)$ & $31(5.2)$ & 0.589 \\
\hline Alcoholism $^{\mathrm{d}}$ & $63(9.0)$ & $11(10.8)$ & $52(8.7)$ & 0.496 \\
\hline Previous surgery & $398(56.9)$ & $53(52.0)$ & $345(57.7)$ & 0.280 \\
\hline Chronic benzodiazepines & $61(8.7)$ & $6(5.9)$ & $55(9.2)$ & 0.273 \\
\hline \multicolumn{5}{|l|}{ Preoperative laboratory tests } \\
\hline Hematocrit $<30 \%$ & $106(15.1)$ & $17(16.7)$ & $89(14.9)$ & 0.642 \\
\hline Albumin $<30 \mathrm{~g} / \mathrm{L}$ & $50(7.1)$ & $8(7.8)$ & $42(7.0)$ & 0.766 \\
\hline Glucose $<4.0$ or $>10.0 \mathrm{mmol} / \mathrm{L}$ & $57(8.1)$ & $4(3.9)$ & $53(8.9)$ & 0.092 \\
\hline $\mathrm{Na}^{+}<135.0$ or $>145.0 \mathrm{mmol} / \mathrm{L}$ & $67(9.6)$ & $9(8.8)$ & $58(9.7)$ & 0.781 \\
\hline $\mathrm{K}^{+}<3.5$ or $>5.5 \mathrm{mmol} / \mathrm{L}$ & $73(10.4)$ & $8(7.8)$ & $65(10.9)$ & 0.355 \\
\hline ASA physical status & & & & 0.130 \\
\hline Class II & $398(56.9)$ & $65(63.7)$ & $333(55.7)$ & \\
\hline Class III & $302(43.1)$ & $37(36.3)$ & $265(44.3)$ & \\
\hline
\end{tabular}

Data are presented as mean $\pm \mathrm{SD}$, number $(\%)$, or median (interquartile range)

$C O P D$ chronic obstructive pulmonary disease, ASA American Society of Anesthesiologists

${ }^{\text {a }}$ Daily smoking of cigarettes up to half a pack for at least 2 years

${ }^{b}$ Alanine aminotransferase and/or aspartate aminotransferase higher than five times of the normal upper limit

${ }^{\mathrm{c}}$ Serum creatinine level $\geq 177 \mu \mathrm{mol} / \mathrm{L}$

${ }^{\mathrm{d}}$ Two drinks or more daily, or weekly consumption of the equivalent of $150 \mathrm{~mL}$ of alcohol

\section{Discussion}

Results of this analysis showed that in elderly patients who were admitted to the ICU after noncardiac surgery, intraoperative handover between anesthesia providers was associated with an increased risk of delirium development after correction for confounding factors.

The development of postoperative delirium results from the complex interaction of a variety of risk factors $[1,9$, 20]. It is estimated that in $30-40 \%$ of cases, postoperative delirium is potentially preventable by reducing the exposure to known risk factors [21, 22]. In addition, some pharmacologic interventions, such as dexmedetomidine, melatonin and antipsychotics, have been investigated for preventing postoperative delirium [15, 23-27]. However, even with the effective prophylactic measures, delirium still occur in some patients [15]. Therefore, further efforts should be made to identify unrecognized risk factors.

With the increasing number and complexity of surgeries [10], handover of anesthesia care is inevitable in clinical practice. In the participating hospitals, day shift anesthesiologists worked from 8 am to $4 \mathrm{pm}$. Between 3 and $4 \mathrm{pm}$, patients of whom the surgeries were continuing were handed over to the next shift anesthesiologists. In addition, patients who underwent surgery beyond $10 \mathrm{pm}$ were handed over to the night shift anesthesiologists. However, studies showed that current practice of intraoperative handover among anesthesia providers is not optimal [14]; and complete handover of intraoperative anesthesia care is associated with worse outcomes, including increased all-cause mortality and more major complications within 30 days after surgery $[13,14]$. 
Table 2 Perioperative variables

\begin{tabular}{|c|c|c|c|c|}
\hline Variable & All patients $(n=700)$ & $\begin{array}{l}\text { With complete handover } \\
(n=102)\end{array}$ & $\begin{array}{l}\text { Without complete hando- } \\
\text { ver }(n=598)\end{array}$ & $P$ value \\
\hline Benzodiazepines at preoperative night & $79(11.3)$ & $12(11.8)$ & $67(11.2)$ & 0.869 \\
\hline Type of anesthesia & & & & 0.233 \\
\hline General & $578(82.6)$ & $80(78.4)$ & $498(83.3)$ & \\
\hline Combined epidural-general & $122(17.4)$ & $22(21.6)$ & $100(16.7)$ & \\
\hline \multicolumn{5}{|l|}{ Intraoperative medication } \\
\hline Nitrous oxide & $523(74.7)$ & 73 (71.6) & $450(75.3)$ & 0.429 \\
\hline Sevoflurane & $501(71.6)$ & $73(71.6)$ & 428 (71.6) & 0.999 \\
\hline Benzodiazepines & $326(46.6)$ & $51(50.0)$ & $275(46.0)$ & 0.453 \\
\hline Propofol & $634(90.6)$ & $97(95.1)$ & $537(89.8)$ & 0.091 \\
\hline Etomidate & $273(39.0)$ & $38(37.3)$ & $235(39.3)$ & 0.696 \\
\hline Glucocorticoids & $646(92.3)$ & $98(96.1)$ & $548(91.6)$ & 0.120 \\
\hline Duration of anesthesia, min & $288(211,386)$ & $416(274,586)$ & $274(202,362)$ & $<0.001$ \\
\hline Duration of surgery, min & $200(126,292)$ & $312(190,479)$ & $189(119,271)$ & $<0.001$ \\
\hline Type of surgery & & & & 0.153 \\
\hline Superficial and transurethral & $69(9.9)$ & $4(3.9)$ & $65(10.9)$ & \\
\hline Intra-abdominal & $475(67.9)$ & $71(69.6)$ & $404(67.6)$ & \\
\hline Intra-thoracic & $120(17.1)$ & $21(20.6)$ & $99(16.5)$ & \\
\hline Spinal and extremital & $36(5.1)$ & $6(5.9)$ & $30(5.0)$ & \\
\hline Grade of surgery ${ }^{a}$ & & & & 0.006 \\
\hline Intermediate & $42(6.0)$ & $0(0.0)$ & $42(7.0)$ & \\
\hline Major or complex & $658(94.0)$ & $102(100.0)$ & $556(93.0)$ & \\
\hline Cardiac risk of surgery ${ }^{b}$ & & & & 0.281 \\
\hline Low & $33(4.7)$ & $2(2.0)$ & $31(5.2)$ & \\
\hline Intermediate & $658(94.0)$ & $98(96.1)$ & $560(93.6)$ & \\
\hline High & $9(1.3)$ & $2(2.0)$ & $7(1.2)$ & \\
\hline Estimated blood loss, ml & $150(50,450)$ & $400(100,800)$ & $100(40,400)$ & $<0.001$ \\
\hline Total intraoperative fluid, $\mathrm{ml}$ & $2510(1600,3700)$ & $3600(2488,5313)$ & $2350(1600,3413)$ & $<0.001$ \\
\hline Intraoperative blood transfusion & $114(16.3)$ & $30(29.4)$ & $84(14.0)$ & $<0.001$ \\
\hline ICU admission with intubation & $382(54.6)$ & $82(80.4)$ & $300(50.2)$ & $<0.001$ \\
\hline Prophylactic dexmedetomidine & $350(50.0)$ & $52(51.0)$ & $298(49.8)$ & 0.830 \\
\hline Postoperative analgesia & & & & 0.051 \\
\hline None & $73(10.4)$ & $5(4.9)$ & $68(11.4)$ & \\
\hline PCIA & $516(73.7)$ & $75(73.5)$ & $441(73.7)$ & \\
\hline PCEA & $111(15.9)$ & $22(21.6)$ & $89(14.9)$ & \\
\hline \multicolumn{5}{|l|}{ Other sedatives/analgesics within 7 days } \\
\hline Propofol & $357(51.0)$ & $80(78.4)$ & $277(46.3)$ & $<0.001$ \\
\hline Benzodiazepines & $58(8.3)$ & $11(10.8)$ & $47(7.9)$ & 0.322 \\
\hline Opioids & $201(28.7)$ & $32(31.4)$ & $169(28.3)$ & 0.521 \\
\hline NSAIDs & $229(32.7)$ & $31(30.4)$ & $198(33.1)$ & 0.589 \\
\hline Pathologically diagnosed cancer & $561(80.1)$ & $84(82.4)$ & 477 (79.8) & 0.545 \\
\hline
\end{tabular}

Data are presented as number (\%), or median (interquartile range)

ICU intensive care unit, PCIA patient-controlled intravenous analgesia, PCEA patient-controlled epidural analgesia, NSAIDs non-steroid antiinflammatory drugs

${ }^{a}$ Rated according to NICE Guidance of Routine preoperative tests for elective surgery [18]

${ }^{\mathrm{b}}$ Rated according to ACC/AHA 2007 guidelines on perioperative cardiovascular evaluation and care for noncardiac surgery [19] 
Fig. 2 Daily prevalence of postoperative delirium in patients with or without complete handover of intraoperative anesthesia care. Some patients died or discharged from hospital within 7 days after surgery, leading to different sample sizes during this period

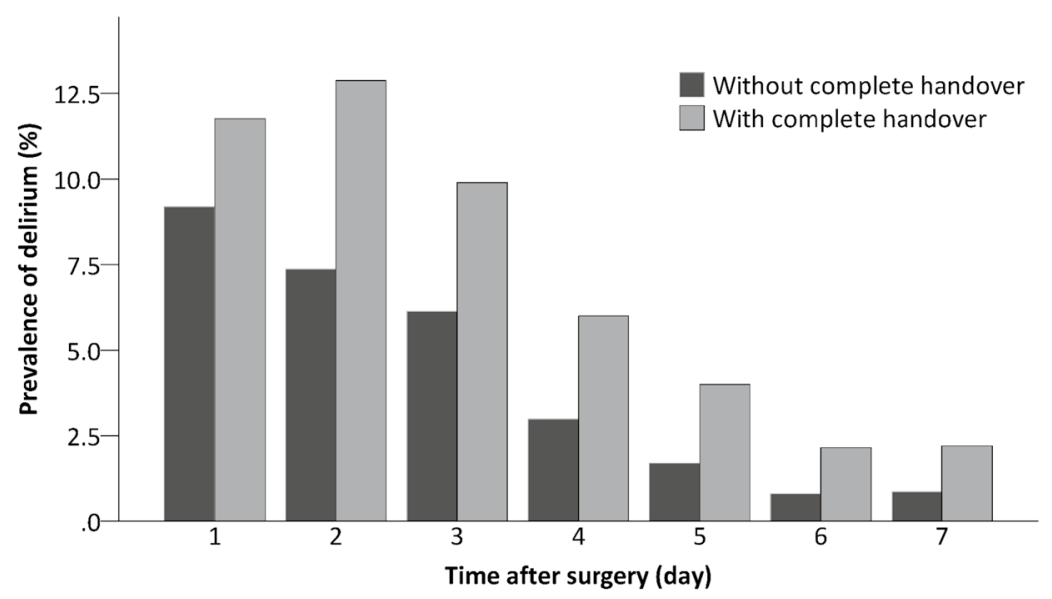

$\begin{array}{rccccccc}\text { Number of Patients } & & & & & & & \\ \text { Without complete handover } & 598 & 597 & 587 & 571 & 530 & 503 & 466 \\ \text { With complete handover } & 102 & 101 & 101 & 100 & 100 & 93 & 91 \\ P \text { values } & 0.415 & 0.062 & 0.161 & 0.217 & 0.271 & 0.237 & 0.255\end{array}$

Table 3 Postoperative outcomes

\begin{tabular}{|c|c|c|c|c|}
\hline Variable & All patients $(n=700)$ & With complete handover $(n=102)$ & $\begin{array}{l}\text { Without complete } \\
\text { handover }(n=598)\end{array}$ & $P$ value \\
\hline Delirium within 7 days & $111(15.9)$ & $23(22.5)$ & $88(14.7)$ & 0.045 \\
\hline Length of stay in ICU, $h$ & $21.1(20.6,21.5)$ & $17.7(16.1,19.3)$ & $21.3(20.9,21.8)$ & 0.336 \\
\hline Time to extubation, $\mathrm{h}$ & $5.2(4.2,6.3)(n=382)$ & $8.7(7.3,10.0)(n=82)$ & $4.5(3.7,5.2)(n=300)$ & 0.054 \\
\hline Non-delirium complications within 30 days & $125(17.9)$ & $29(28.4)$ & $96(16.1)$ & 0.003 \\
\hline Length of stay in hospital after surgery, day & $11.0(10.4,11.6)$ & $14.0(12.0,16.0)$ & $10.0(9.4,10.6)$ & 0.001 \\
\hline All-cause 30-day mortality & $5(0.7)$ & $2(2.0)$ & $3(0.5)$ & 0.156 \\
\hline
\end{tabular}

Data are presented as number $(\%)$ or median (95\% confidence interval)

$I C U$ intensive care unit

For the first time, our results showed that intraoperative handover of anesthesia care was associated with an increased risk of postoperative delirium. Reasons leading to this result may include the following. First, intraoperative handover of patients' information might be insufficient. This was reported by previous studies [14]. Given that the incoming doctors did not completely understand patients' condition, anesthetic management might be suboptimal during subsequent surgery and led to increased delirium. Second, at ICU admission, the handover of patients' information might be further comprised between the successive anesthesiologists and the ICU physicians. This might have led to increased complications including delirium [28]. Third, patients with complete handover usually had their surgeries ended late and returned to the ward late. It was possible that this worsened patients' sleep quality and increased delirium, as poor sleep quality is associated with increased delirium [29]. At last, patients required handover of anesthesia care usually underwent long-duration surgery, which might also increase delirium [30, 31]. However, the association between surgical duration and delirium development was not found in our results.

Our results also found that patients with complete handover had a higher incidence of non-delirium complications and stayed longer in hospital after surgery. These were in line with previously reported results [13]. On the other hand, a good handover of patients between anesthesiologists and intensive care unit physicians can be achieved using a protocol or checklist $[32,33]$, and is associated with improved outcomes including less unplanned extubation and shortened ventilation times [34]. However, whether good intraoperative handover among anesthesia providers can decrease delirium and improve outcomes has not been well investigated.

There are several limitations of this secondary analysis. First, in the original trial, only patients who were admitted to the ICU after surgery were recruited and half of them received prophylactic dexmedetomidine. These limited the generalisability of our results and might produce bias by 


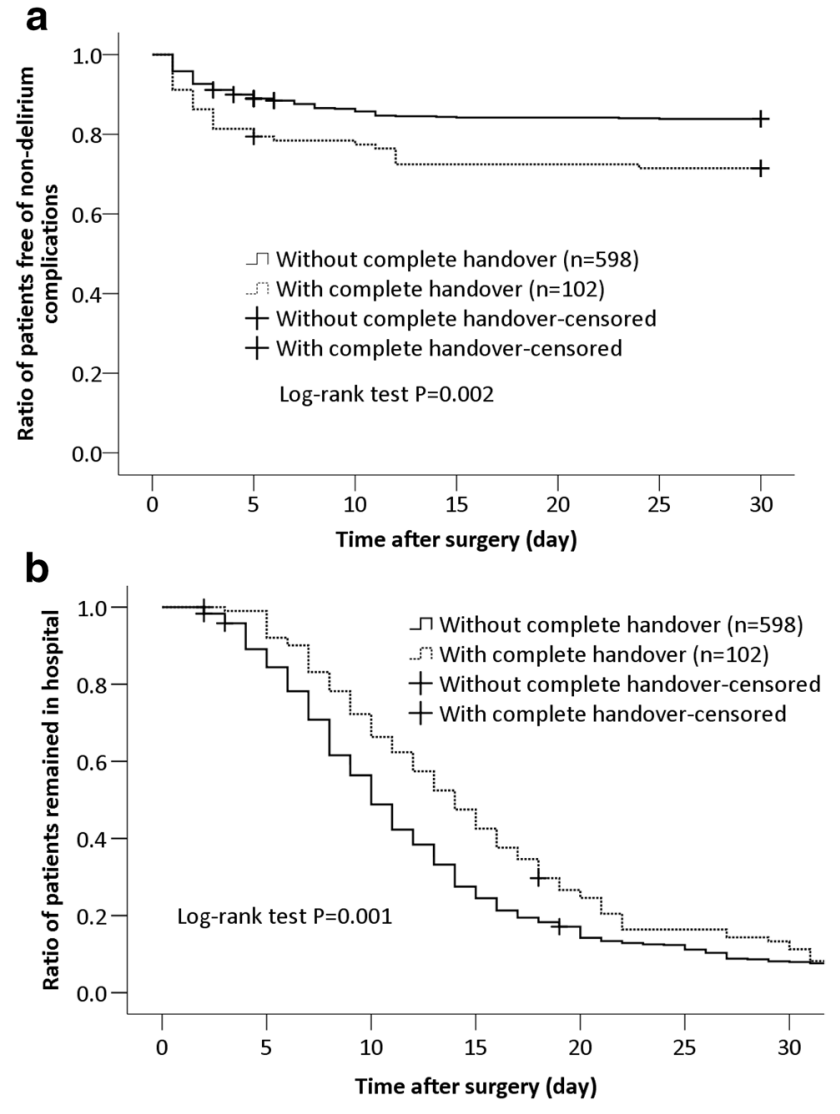

Fig. 3 The occurrence of non-delirium complications (a) and the length of stay in hospital after surgery $(\mathbf{b})$ in patients with or without complete handover of intraoperative anesthesia care reducing postoperative delirium. Second, patients with or without complete handover exhibited differences in some baseline and perioperative variables which might affect delirium development. Although a multivariate regression model was used to adjust for confounding factors, we cannot completely exclude the interference of these factors. Lastly, we cannot establish a causal relationship between handover of anesthesia care and development of postoperative delirium from a secondary analysis. However, our results provide clues for further interventional studies.

\section{Conclusions}

Our results indicated that intraoperative handover of anesthesia care was associated with an increased risk of delirium development in elderly patients admitted to ICU after noncardiac surgery. Considering our results and others, studies investigating measures to improve intraoperative handover are urgently needed.

Acknowledgements The authors gratefully acknowledge Prof. Xin-Yu Sun (Psychiatric Department, Peking University Sixth Hospital, Beijing, China) for her help in psychiatric consultation.

Funding The original study was supported by Braun Anesthesia Scientific Research Fund and Wu Jieping Medical Foundation, Beijing, China.
Table 4 Factors in association with postoperative delirium

\begin{tabular}{|c|c|c|c|c|}
\hline \multirow[t]{2}{*}{ Variable } & \multicolumn{2}{|l|}{ Univariate analyses $^{\mathrm{a}}$} & \multicolumn{2}{|l|}{ Multivariate analysis $^{\mathrm{b}}$} \\
\hline & OR $(95 \% \mathrm{CI})$ & $P$ value & OR $(95 \% \mathrm{CI})$ & $P$ value \\
\hline Complete handover of anesthesia care & $1.687(1.006-2.828)$ & 0.041 & $1.787(1.012-3.155)$ & 0.046 \\
\hline Age, years & $1.049(1.019-1.081)$ & 0.001 & $1.041(1.008-1.074)$ & 0.013 \\
\hline Body mass index, $\mathrm{kg} / \mathrm{m}^{2}$ & $0.902(0.853-0.954)$ & $<0.001$ & $0.930(0.878-0.985)$ & 0.013 \\
\hline Previous stroke & $1.692(1.083-2.644)$ & 0.021 & $1.785(1.104-2.886)$ & 0.018 \\
\hline Preoperative albumin $<30 \mathrm{~g} / \mathrm{L}$ & $2.473(1.301-4.702)$ & 0.006 & $1.700(0.838-3.450)$ & 0.142 \\
\hline Intraoperative use of etomidate & $1.823(1.212-2.742)$ & 0.004 & $1.373(0.879-2.143)$ & 0.163 \\
\hline ICU admission with intubation & $2.109(1.365-3.257)$ & 0.001 & $1.859(1.162-2.974)$ & 0.010 \\
\hline Prophylactic dexmedetomidine ${ }^{c}$ & $0.345(0.222-0.537)$ & $<0.001$ & $0.354(0.223-0.560)$ & $<0.001$ \\
\hline Postoperative propofol within 7 days ${ }^{\mathrm{d}}$ & $1.570(1.038-2.373)$ & 0.032 & - & - \\
\hline Pathologically diagnosed cancer & $0.519(0.328-0.820)$ & 0.005 & $0.610(0.369-1.007)$ & 0.053 \\
\hline
\end{tabular}

${ }^{\text {a }}$ Postoperative delirium within 7 days was modeled as a function of a single factor in the univariate logistic regression analyses

${ }^{\text {b}}$ Postoperative delirium within 7 days was modeled as a function of all factors with a $P$ value $<0.05$ in the univariate analyses. Multivariate Logistic regression analysis was performed using a Enter procedure. Hosmer-Lemeshow test of goodness of fit of the model: $\chi^{2}=7.651, d f=8, P=0.468$

${ }^{c}$ Administered as a continuous infusion at a rate of $0.1 \mu \mathrm{g} / \mathrm{kg} / \mathrm{h}$ from ICU admission to 8 am of next morning [15]

${ }^{\mathrm{d}}$ Not included in multivariable analysis because of correlation with ICU admission with intubation 


\section{Compliance with ethical standards}

Conflict of interest Dong-Xin Wang reports lecture fees and travel expenses for lectures given at domestic academic meetings from Jiangsu Hengrui Medicine Co Ltd, China, and Yichang Humanwell Pharmaceutical Co Ltd, China. The other authors report no conflicting interests.

Open Access This article is distributed under the terms of the Creative Commons Attribution 4.0 International License (http://creativeco mmons.org/licenses/by/4.0/), which permits unrestricted use, distribution, and reproduction in any medium, provided you give appropriate credit to the original author(s) and the source, provide a link to the Creative Commons license, and indicate if changes were made.

\section{References}

1. Scholz AF, Oldroyd C, McCarthy K, Quinn TJ, Hewitt J. Systematic review and meta-analysis of risk factors for postoperative delirium among older patients undergoing gastrointestinal surgery. Br J Surg. 2016;103(2):e21-8. https://doi.org/10.1002/bjs.10062.

2. Aldecoa C, Bettelli G, Bilotta F, Sanders RD, Audisio R, Borozdina A, Cherubini A, Jones C, Kehlet H, MacLullich A, Radtke F, Riese F, Slooter AJ, Veyckemans F, Kramer S, Neuner B, Weiss B, Spies CD. European Society of Anaesthesiology evidence-based and consensus-based guideline on postoperative delirium. Eur J Anaesthesiol. 2017;34(4):192-214. https://doi. org/10.1097/EJA.0000000000000594.

3. Aitken SJ, Blyth FM, Naganathan V. Incidence, prognostic factors and impact of postoperative delirium after major vascular surgery: a meta-analysis and systematic review. Vasc Med. 2017;22(5):387-97. https://doi.org/10.1177/1358863X17721639.

4. Yang Y, Zhao X, Dong T, Yang Z, Zhang Q, Zhang Y. Risk factors for postoperative delirium following hip fracture repair in elderly patients: a systematic review and meta-analysis. Aging Clin Exp Res. 2017;29(2):115-26. https://doi.org/10.1007/s4052 0-016-0541-6.

5. Abelha FJ, Luís C, Veiga D, Parente D, Fernandes V, Santos P, Botelho M, Santos A, Santos C. Outcome and quality of life in patients with postoperative delirium during an ICU stay following major surgery. Crit Care. 2013;17(5):R257. https://doi. org/10.1186/cc13084.

6. Oh ES, Sieber FE, Leoutsakos JM, Inouye SK, Lee HB. Sex differences in hip fracture surgery: preoperative risk factors for delirium and postoperative outcomes. J Am Geriatr Soc. 2016;64(8):161621. https://doi.org/10.1111/jgs.14243.

7. Stachon P, Kaier K, Zirlik A, Reinöhl J, Heidt T, Bothe W, Hehn $\mathrm{P}$, Zehender M, Bode C. Von Zur Mühlen C. Risk factors and outcome of postoperative delirium after transcatheter aortic valve replacement. Clin Res Cardiol. 2018;107(9):756-62. https://doi. org/10.1007/s00392-018-1241-3.

8. Galyfos GC, Geropapas GE, Sianou A, Sigala F, Filis K. Risk factors for postoperative delirium in patients undergoing vascular surgery. J Vasc Surg. 2017;66(3):937-46. https://doi.org/10.1016/j. jvs.2017.03.439.

9. Reddy SV, Irkal JN, Srinivasamurthy A. Postoperative delirium in elderly citizens and current practice. J Anaesthesiol Clin Pharmacol. 2017;33(3):291-9. https://doi.org/10.4103/joacp.JOACP -180_16.

10. Weiser TG, Haynes AB, Molina G, Lipsitz SR, Esquivel MM, Uribe-Leitz T, Fu R, Azad T, Chao TE, Berry WR, Gawande AA. Size and distribution of the global volume of surgery in 2012. Bull
World Health Organ. 2016;94(3):201-9f. https://doi.org/10.2471/ blt.15.159293.

11. White MS, Burns C, Conlon HA. The impact of an aging population in the workplace. Workplace Health Saf. 2018;66(10):493-8. https://doi.org/10.1177/2165079917752191.

12. Hudson CC, McDonald B, Hudson JK, Tran D, Boodhwani M. Impact of anesthetic handover on mortality and morbidity in cardiac surgery: a cohort study. J Cardiothorac Vasc Anesth. 2015;29(1):11-6. https://doi.org/10.1053/j.jvca.2014.05.018.

13. Jones PM, Cherry RA, Allen BN, Jenkyn KMB, Shariff SZ, Flier S, Vogt KN, Wijeysundera DN. Association between handover of anesthesia care and adverse postoperative outcomes among patients undergoing major surgery. JAMA. 2018;319(2):143-53. https://doi.org/10.1001/jama.2017.20040.

14. Choromanski D, Frederick J, McKelvey GM, Wang H. Intraoperative patient information handover between anesthesia providers. J Biomed Res. 2014;28(5):383-7. https://doi.org/10.7555/ JBR.28.20140001.

15. Su X, Meng ZT, Wu XH, Cui F, Li HL, Wang DX, Zhu X, Zhu SN, Maze M, Ma D. Dexmedetomidine for prevention of delirium in elderly patients after non-cardiac surgery: a randomised, double-blind, placebo-controlled trial. Lancet. 2016;388(10054):1893-902. https://doi.org/10.1016/S0140 $-6736(16) 30580-3$.

16. Ely EW, Inouye SK, Bernard GR, Gordon S, Francis J, May L, Truman B, Speroff T, Gautam S, Margolin R, Hart RP, Dittus R. Delirium in mechanically ventilated patients: validity and reliability of the confusion assessment method for the intensive care unit (CAM-ICU). JAMA. 2001;286(21):2703-10. https://doi. org/10.1001/jama.286.21.2703.

17. Sessler CN, Gosnell MS, Grap MJ, Brophy GM, O’Neal PV, Keane KA, Tesoro EP, Elswick RK. The Richmond AgitationSedation Scale: validity and reliability in adult intensive care unit patients. Am J Respir Crit Care Med. 2002;166(10):1338-44. https://doi.org/10.1164/rccm.2107138.

18. NICE Guidance Routine preoperative tests for elective surgery. BJU Int. 2018;121(1):12-6. https://doi.org/10.1111/bju.14079.

19. Fleisher LA, Beckman JA, Brown KA, Calkins H, Chaikof E, Fleischmann KE, Freeman WK, Froehlich JB, Kasper EK, Kersten JR, Riegel B, Robb JF, Smith SC Jr, Jacobs AK, Adams CD, Anderson JL, Antman EM, Buller CE, Creager MA, Ettinger SM, Faxon DP, Fuster V, Halperin JL, Hiratzka LF, Hunt SA, Lytle BW, Nishimura R, Ornato JP, Page RL, Tarkington LG, Yancy $\mathrm{CW}$, American College of Cardiology/American Heart Association Task Force on Practice Guidelines, American Society of Echocardiography, American Society of Nuclear Cardiology, Heart Rhythm Society, Society of Cardiovascular Anesthesiologists, Society for Cardiovascular Angiography and Interventions, Society for Vascular Medicine and Biology, Society for Vascular Surgery. ACC/AHA 2007 guidelines on perioperative cardiovascular evaluation and care for noncardiac surgery: a report of the American College of Cardiology/American Heart Association Task Force on Practice Guidelines (Writing Committee to Revise the 2002 Guidelines on Perioperative Cardiovascular Evaluation for Noncardiac Surgery): developed in collaboration with the American Society of Echocardiography, American Society of Nuclear Cardiology, Heart Rhythm Society, Society of Cardiovascular Anesthesiologists, Society for Cardiovascular Angiography and Interventions, Society for Vascular Medicine and Biology, and Society for Vascular Surgery. Circulation. 2007;116(17):e418500. https://doi.org/10.1161/CIRCULATIONAHA.107.185699.

20. Raats JW, Steunenberg SL, de Lange DC, van der Laan L. Risk factors of post-operative delirium after elective vascular surgery in the elderly: a systematic review. Int J Surg. 2016;35:1-6. https ://doi.org/10.1016/j.ijsu.2016.09.001. 
21. Inouye SK, Bogardus ST Jr, Charpentier PA, Leo-Summers L, Acampora D, Holford TR, Cooney LM Jr. A multicomponent intervention to prevent delirium in hospitalized older patients. $\mathrm{N}$ Engl J Med. 1999;340(9):669-76. https://doi.org/10.1056/nejm1 99903043400901.

22. Martocchia A, Curto M, Comite F, Scaccianoce S, Girardi P, Ferracuti S, Nicoletti F, Falaschi P, Orthogeriatric Group. The prevention and treatment of delirium in elderly patients following hip fracture surgery. Recent Pat CNS Drug Discov. 2015;10(1):55-64. https://doi.org/10.2174/1574889810666150216152624.

23. Deiner S, Luo X, Lin HM, Sessler DI, Saager L, Sieber FE, Lee HB, Sano M, The Dexlirium Writing Group, Jankowski C, Bergese SD, Candiotti K, Flaherty JH, Arora H, Shander A, Rock P. Intraoperative infusion of dexmedetomidine for prevention of postoperative delirium and cognitive dysfunction in elderly patients undergoing major elective noncardiac surgery: a randomized clinical trial. JAMA Surg. 2017;152(8):e171505. https:// doi.org/10.1001/jamasurg.2017.1505.

24. Hanania M, Kitain E. Melatonin for treatment and prevention of postoperative delirium. Anesth Analg. 2002;94(2):338-9. https:// doi.org/10.1213/00000539-200202000-00019.

25. Larsen KA, Kelly SE, Stern TA, Bode RH Jr, Price LL, Hunter DJ, Gulczynski D, Bierbaum BE, Sweeney GA, Hoikala KA, Cotter JJ, Potter AW. Administration of olanzapine to prevent postoperative delirium in elderly joint-replacement patients: a randomized, controlled trial. Psychosomatics. 2010;51(5):409-18. https://doi. org/10.1176/appi.psy.51.5.409.

26. Seitz D, Gill SS. Perioperative haloperidol to prevent postoperative delirium. J Am Geriatr Soc. 2006;54(5):861. https://doi.org/ 10.1111/j.1532-5415.2006.00714.x.

27. Fok MC, Sepehry AA, Frisch L, Sztramko R, Borger van der Burg BL, Vochteloo AJ, Chan P. Do antipsychotics prevent postoperative delirium? A systematic review and meta-analysis. Int J Geriatr Psychiatry. 2015;30(4):333-44. https://doi.org/10.1002/gps.4240.

28. Bonifacio AS, Segall N, Barbeito A, Taekman J, Schroeder R, Mark JB. Handovers from the OR to the ICU. Int Anesthesiol Clin. 2013;51(1):43-61. https://doi.org/10.1097/AIA.0b013e3182 $6 f 2 b 0 e$.
29. Su X, Wang DX. Improve postoperative sleep: what can we do? Curr Opin Anaesthesiol. 2018;31(1):83-8. https://doi. org/10.1097/ACO.0000000000000538.

30. Cereghetti C, Siegemund M, Schaedelin S, Fassl J, Seeberger MD, Eckstein FS, Steiner LA, Goettel N. Independent predictors of the duration and overall burden of postoperative delirium after cardiac surgery in adults: an observational cohort study. J Cardiothorac Vasc Anesth. 2017;31(6):1966-73. https://doi.org/10.1053/j. jvca.2017.03.042.

31. Nazemi AK, Gowd AK, Carmouche JJ, Kates SL, Albert TJ, Behrend CJ. Prevention and management of postoperative delirium in elderly patients following elective spinal surgery. Clin Spine Surg. 2017;30(3):112-9. https://doi.org/10.1097/ BSD.0000000000000467.

32. Petrovic MA, Aboumatar H, Baumgartner WA, Ulatowski JA, Moyer J, Chang TY, Camp MS, Kowalski J, Senger CM, Martinez EA. Pilot implementation of a perioperative protocol to guide operating room-to-intensive care unit patient handoffs. J Cardiothorac Vasc Anesth. 2012;26(1):11-6. https://doi.org/10.1053/j. jvca.2011.07.009.

33. Dixon JL, Stagg HW, Wehbe-Janek H, Jo C, Culp WC Jr, Shake JG. A standard handoff improves cardiac surgical patient transfer: operating room to intensive care unit. J Healthc Qual. 2015;37(1):22-32. https://doi.org/10.1097/01.JHQ.0000460123 .91061.b3.

34. Kaufmnan J, Twite M, Barrett C, Peyton C, Koehler J, Rannie M, Kahn MG, Schofield S, Ing RJ, Jaggers J, Hyman D, da Cruz EM. A handoff protocol from the cardiovascular operating room to cardiac ICU is associated with improvements in care beyond the immediate postoperative period. Jt Comm J Qual Patient Saf. 2013;39(7):306-11. https://doi.org/10.1016/s1553 $-7250(13) 39043-6$.

Publisher's Note Springer Nature remains neutral with regard to jurisdictional claims in published maps and institutional affiliations. 